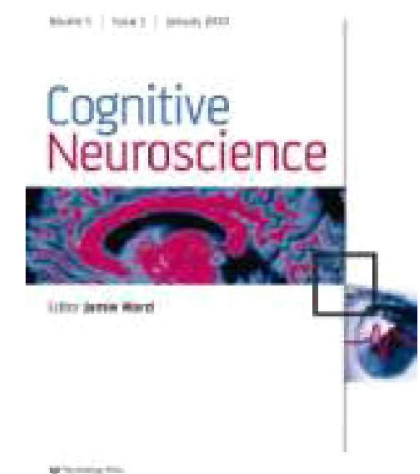

\title{
Ventral and dorsal streams as modality-independent phenomena
}

\begin{tabular}{|c|c|}
\hline Journal: & Cognitive Neuroscience \\
\hline Manuscript ID: & pCNS-2009-CM-0046.R2 \\
\hline Manuscript Type: & Commentary \\
\hline $\begin{array}{r}\text { Date Submitted by the } \\
\text { Author: }\end{array}$ & \\
\hline Complete List of Authors: & $\begin{array}{l}\text { Dyson, Ben; Ryerson University, Psychology Department } \\
\text { Dunn, Andrew; Nottingham Trent University } \\
\text { Alain, Claude; The Rotman Research Institute }\end{array}$ \\
\hline Keywords: & dorsal and ventral streams, dual pathway, auditory scene analysis \\
\hline
\end{tabular}

\section{s) scholaroNE" \\ Manuscript Central}


Ventral and dorsal streams as modality-independent phenomena

\section{Benjamin J. Dyson}

Department of Psychology, Ryerson University, Canada.

Andrew K. Dunn

Division of Psychology, Nottingham Trent University, UK

Claude Alain

The Rotman Research Institute, Canada.
Telephone : (001) 416-979-5000

Fax : $\quad$ (001) 416-979-5273

E-mail : ben.dyson@ psych.ryerson.ca 


\begin{abstract}
Interest in ventral and dorsal streams is not limited to vision and the functionality of similar pathways in other domains has also been considered. Auditory dual pathway models share many conceptual and empirical concerns with those put forward for vision including the absolute versus relative, localized versus distributed, and the exact nature of functionality of the two streams. Despite their problems, dual pathway hypotheses provide broad frameworks with which to consider cortical architecture across the senses.
\end{abstract}


Schenk and McIntosh reject a strong version of visual ventral and dorsal activity, in which perception (ventral) and action (dorsal) are viewed as functionally independent. Dual pathway models of visual processing have had a tremendous impact on cognitive neurosciences and have recently been extended to account for auditory processing (Kaas \& Hackett, 1999; also tactile processing, Dijkerman \& De Haan, 2007). Given the opportunity for massive cortical interconnectivity, it seems unlikely that spatially-distributed and temporally-coordinated processing streams covering large areas within the brain will show complete functional independence in any modality. However, weaker versions of auditory ventral and dorsal activity have been considered in which certain processes tend to be carried out along one pathway rather than another.

Despite the perception-action distinction being the current dominant force in the characterization of visual ventral and dorsal activity, the older what-where distinction has been the focus of much of the auditory literature. This was recently summarized in a functional neuroimaging metaanalysis examining ventral and dorsal activity across 36 studies (Arnott, Binns, Grady \& Alain, 2004). Equivalent degrees of initial activation for spatial (where) and non-spatial (what) information were found in the posterior temporal lobe, which then partitioned into a dorsal route to the superior frontal sulcus in which spatial tasks generated greater activity, and, a ventral route to the inferior frontal gyrus in which non-spatial tasks generated greater activity. Further evidence for a what/where distinction is provided by neurological case studies, in which patients collectively show a double dissociation between lesion profile and performance, namely problems in sound localization and damage to more posterior / parietal areas, and problems in sound identification and damage to more lateral / temporal areas (Clarke, Bellmann, Meuli, Assal 
\& Steck, 2000). Consistent with the weak version of visual ventral and dorsal activity, these paths are best characterized by relative rather than absolute differences in the flow of certain kinds of information and operation.

In a transition similar to that observed in the visual literature, discussion of ventral (what) and dorsal (where) processing in audition has begun to focus on multiple interpretations of the dorsal stream, while the ventral stream continues to be associated with stimulus identity. Alternative conceptualizations have recast the auditory dorsal stream as a pathway critical for sensory-motor integration and goal-directed (speech) action (do pathway; Warren, Wise, \& Warren, 2005), and, as a pathway critical for the processing of spectral motion: how an auditory signal changes over time (also known a 'how' pathway; Belin \& Zatorre, 2000). Emphasizing the importance of auditory time rather than space in the dorsal stream may be a reasonable maneuver in terms of the proposed preference for spatial aspects of visual processing, and temporal aspects of auditory processing (e.g., Kubovy \& Van Valkenburg, 2001).

Ultimately the degree of localized functionality must be tempered by the acknowledgement that dorsal and ventral streams have numerous opportunities to communicate with one another both during auditory (Hall, 2003) and visual (Milner \& Goodale, 1995) processing. Such observations make a weak version of the ventral/dorsal model more likely, highlighting the need to understand where and when inter-stream communications arise. Despite the attraction of sensory isomorphism, the eventual preferences of such neural trajectories may rest with the architecture of individual sensory systems, the specific task demands (Glover, 2002), and, also the eventual need to integrate information across modalities in pursuit of a multi-sensory environment in 
which we may operate successfully. Despite its flaws, the ventral / dorsal distinction provides us with a framework with which to explore these issues.

1

2

3

4

5

6

7

8

9

10

11

12

13

14

15

16

17

18

19

20

21

22

23

24

25

26

27

28

29

30

31

32

33

34

35

36

37

38

39

40

41

42

43

44

45

46

47

48

49

50

51

52

53

54

55

56

57

58

59

60 
Arnott, S. R., Binns , M. A., Grady , C. L. , and Alain , C. (2004). Assessing the auditory dualpathway model in humans. Neuroimage, 22 401-8.

Belin , P. and Zatorre, R. J. (2000). 'What', 'where' and 'how' in auditory cortex. Nature Neuroscience, 3, 965-6.

Clarke, S., Bellmann, A., Meuli, R. A., Assal, G. \& Steck, A. J. (2000). Auditory agnosia and auditory spatial deficits following left hemispheric lesions: Evidence for distinct processing pathways. Neuropsychologia, 38, 797-807.

Dijkerman, H. C., \& De Haan, E. H. F. (2007). Somatosensory processes subserving perception and action. Behavioral and Brain Sciences, 30, 189-201.

Glover, S. (2002). Visual illusion affect planning but not control. Trends in Cognitive Sciences, 6, 288-292.

Hall , D. A. (2003). Auditory pathways: are 'what' and 'where' appropriate? Current Biology, 13, R406-8.

Kaas, J. H. and Hackett, T. A. (1999). 'What' and 'where' processing in auditory cortex. Nature Neuroscience, 2, 1045-7.

Kubovy, M. and Van Valkenburg, D. (2001). Auditory and visual objects. Cognition, 80, $97-$ 126.

Milner, A. D. \& Goodale, M. A. (1995). The visual brain in action. Oxford University Press: Oxford.

Warren, J. E., Wise, R. J. S. \& Warren, J. D. (2005). Sounds do-able: auditory-motor transformations and the posterior temporal plane. Trends In Neurosciences, 28, 636-643. 